Article type : Mini commentary

Mini-commentary on BJOG- 19-1590.R1: Obstetric outcomes of twin pregnancies presenting with a complete hydatidiform mole and coexistent normal fetus: A systematic review and metaanalysis

\title{
Vesicular mole in a twin pregnancy
}

Amarnath Bhide

Fetal Medicine Unit

St George's University Hospitals NHS Foundation Trust

London

Email: abhide@sgul.ac.uk

The authors describe the problems associated with a twin pregnancy combining a complete vesicular mole and an apparently normal fetus (BJOG $2020 \mathrm{xxxx}$ ). The problem is uncommon and the diagnosis may prove challenging. The ultrasound features of this condition can be very similar to those of placental mesenchymal dysplasia (PMD) or a partial vesicular mole.

PMD is an under-recognised entity. The most common sonographic features include enlarged (50\%) and cystic (80\%) placenta in the first half of pregnancy and dilated chorionic vessels later on (Nayeri et al, Ultrasound Obstet Gynecol, 2013). Elevated maternal serum hCG and AFP levels are common. Association with imprinting disorders (particularly Beckwith-Wiedemann syndrome) in the fetus has been reported. Therefore, careful search for features of this syndrome (enlarged cystic kidneys, exomphalos, glossoptosis) is warranted. Trophoblastic proliferation and stromal inclusions are not seen in PMD, and the risk of gestational trophoblastic neoplasia is minimal. Paternal uniparental disomy of two key genes has been reported in PMD (Robinson et al, Hum Reprod 2007). In complete hydatidiform mole the whole genome is

This article has been accepted for publication and undergone full peer review but has not been through the copyediting, typesetting, pagination and proofreading process, which may lead to differences between this version and the Version of Record. Please cite this article as doi: $\underline{10.1111 / 1471-0528.16325}$

This article is protected by copyright. All rights reserved 
affected with uniparental disomy, explaining the similar ultrasound features. Partial vesicular mole is much less common. The placenta is triploid as well as the fetus. Cases with a normal fetus result from confined placental mosaicism where the placenta is triploid but the fetus diploid (Kawasaki et al, JOGR, 2016).

After making a diagnosis of complete vesicular mole and an apparently normal fetus in a twin pregnancy using ultrasound, invasive prenatal diagnosis (amniocentesis) should be considered. Molecular diagnosis of Beckwith Wiedemann syndrome on the amniotic fluid sample should be offered. However, the parents should be informed that the sensitivity of molecular diagnosis is only $80 \%$. From the current review, we learn that the obstetric outlook is guarded for a twin pregnancy consisting of a complete vesicular mole and an apparently normal fetus. It is not any less problematic for the other possibilities though. Even if invasive prenatal diagnosis results are apparently normal in cases of PMD or a partial vesicular mole with apparently normal fetus, the risks of growth restriction, stillbirth and pre-eclampsia remain high. We learn that gestational trophoblastic neoplasia (GTN) develops in about a third of all twin pregnancies combining a complete vesicular mole and an apparently normal fetus, even with voluntary pregnancy termination. This underscores the need of a close surveillance regardless of the outcome of the pregnancy.

Another peculiar problem that we need to keep in mind is the possibility of a falsely negative test for beta hCG in this condition. hCG molecules can completely saturate the binding sites of the sandwich radio-immunoassay, the typical assay used for estimation of maternal serum or urinary beta hCG and falsely lead to a negative result. This phenomenon, 'The hook effect', has been reported with levels in excess of 1000000 IU/L (Tabas et al, N Engl J Med 2003). Appropriate prior dilution (at least 1:10) of the specimen would avoid this problem. Therefore, it is important to alert the laboratory about the possible diagnosis.

No disclosures: A completed disclosure of interest form is available to view online as supporting information.

This article is protected by copyright. All rights reserved 